\title{
Article
}

\section{A fixed point theorem for generalized weakly contractive mappings in $b$-metric spaces}

\author{
Eliyas Zinab ${ }^{1}$, Kidane Koyas ${ }^{1}$ and Aynalem Girma ${ }^{1, *}$ \\ 1 Department of Mathematics, College of Natural Sciences, Jimma University, Jimma, Ethiopia.; \\ eliyaszinab7@gmail.com (E.Z); kidanekoyas@yahoo.com (K.K); aynalemgirma46@yahoo.com (A.G) \\ * Correspondence: aynalemgirma46@yahoo.com
}

Received: 11 July 2019; Accepted: 30 September 2019; Published: 16 January 2020.

\begin{abstract}
In this paper we establish a fixed point theorem for generalized weakly contractive mappings in the setting of $b$-metric spaces and prove the existence and uniqueness of a fixed point for a self-mappings satisfying the established theorem. Our result extends and generalizes the result of Cho [1]. Finally, we provided an example in the support of our main result.
\end{abstract}

Keywords: Fixed point, generalized weak contractive mapping, $b$-metric space.

MSC: 26D10, 31A10, 26A33.

\section{Introduction}

I

n 1993, Czerwik [2] introduced the concept of $b$-metric spaces and proved the Banach contraction mapping principle in the setting of $b$-metric spaces. Afterwards, several research papers [3-8] were published on the existence of fixed point results for single-valued and multi-valued mappings in the setting of b-metric spaces. In 1997, Alber et al. [9] generalized Banach's contraction principle by introducing the concept of weakly contractive mappings and proved the existence of fixed points for weakly contractive and single valued mappings on Hilbert spaces.

Rhoades [10] proved that every weakly contractive mapping has a unique fixed point in complete metric spaces. Then, many authors obtained generalizations and extensions of the weakly contractive mappings.

In particular, Choudhury et al. [11] generalized fixed point results for weakly contractive mappings by using altering distance functions. Very recently, Cho [1] introduced the notion of generalized weakly contractive mappings in metric spaces and proved a fixed point theorem for generalized weakly contractive mappings defined on complete metric spaces.

Inspired and motivated by the results of Cho [1] the purpose of this paper is to establish a fixed point result for generalized weakly contractive mappings in the setting of $b$-metric spaces.

\section{Preliminaries}

In this section, we give basic definitions of concepts concerning a generalized weakly contractive mappings in the setting of $b$-metric spaces.

Definition 1. [2] Let $X$ be a nonempty set and $s \geq 1$ be a given real number. A function $d: X \times X \rightarrow R^{+}$is a $b$-metric if and only if for all $x, y, z \in X$, the following conditions are satisfied:

(a) $d(x, y)=0$ if and only if $x=y$;

(b) $d(x, y)=d(y, x)$;

(c) $d(x, z) \leq s[d(x, y)+d(y, z)]$.

The pair $(X, d)$ is called a $b$-metric space.

It should be noted that the class of $b$-metric spaces is effectively larger than that of metric spaces, since $b$-metric is metric when $s=1$. But, in general, the converse is not true. 
Example 1. [12] Let $X=R$ and $d: X \times X \rightarrow R^{+}$be given by $d(x, y)=(x-y)^{2}$ for all $x, y \in X$, then $d$ is a $b$-metric on $X$ with $s=2$ but it is not a metric on $X$, because for $x=2, y=4$ and $z=6$, we have $d(2,6) \not \leq 2[d(2,4)+d(4,6)]$, hence the triangle inequality for a metric does not hold.

Definition 2. A function $f: X \rightarrow R^{+}$, where $X$ is $b$-metric space is called lower semicontinuous if for all $x \in X$ and $x_{n} \in X$ with $\lim _{n \rightarrow \infty} x_{n}=x$, we have

$$
f(x) \leq \liminf _{n \rightarrow \infty} f\left(x_{n}\right) .
$$

Definition 3. [6] Let $X$ be a $b$-metric space and $\left\{x_{n}\right\}$ be a sequence in $X$, we say that

(a) $x_{n}$ is $b$-converges to $x \in X$ if $d\left(x_{n}, x\right) \rightarrow 0$ as $n \rightarrow \infty$.

(b) $x_{n}$ is a $b$-Cauchy sequence if $d\left(x_{n}, x_{m}\right) \rightarrow 0$ as $n, m \rightarrow \infty$.

(c) $(X, d)$ is $b$-complete if every $b$-Cauchy sequence in $X$ is $b$-convergent.

Definition 4. [1] Let $X$ be a complete metric space with metric $d$, and $T: X \rightarrow X$. Also let $\varphi: X \rightarrow R^{+}$be a lower semicontinuous function, then $T$ is called a generalized weakly contractive mapping if it satisfies the following condition:

$$
\psi(d(T x, T y)+\varphi(T x)+\varphi(T y)) \leq \psi(m(x, y, d, T, \varphi))-\phi(l(x, y, d, T, \varphi))
$$

where,

$$
\begin{aligned}
m(x, y, d, T, \varphi)= & \max \{d(x, y)+\varphi(x)+\varphi(y), d(x, T x)+\varphi(x)+\varphi(T x), d(y, T y)+\varphi(y)+\varphi(T y), \\
& \left.\frac{1}{2}[d(x, T y)+\varphi(x)+\varphi(T y)+d(y, T x)+\varphi(y)+\varphi(T x)]\right\}
\end{aligned}
$$

and $l(x, y, d, T, \varphi)=\max \{d(x, y)+\varphi(x)+\varphi(y), d(y, T y)+\varphi(y)+\varphi(T y)\}$, for all $x, y \in X$, where $\psi: R^{+} \rightarrow R^{+}$ is a continuous with $\psi(t)=0$ if and only if $t=0$ and $\phi: R^{+} \rightarrow R^{+}$is a lower semicontinuous function with $\phi(t)=0$ if and only if $t=0$.

Theorem 1. [1] Let $X$ be complete. If $T$ is a generalized weakly contractive mapping, then there exists a unique $z \in X$ such that $z=T z$ and $\varphi(z)=0$.

Lemma 1. [12] Suppose $(X, d)$ is a b-metric space and $\left\{x_{n}\right\}$ be a sequence in $X$ such that

$$
\lim _{n \rightarrow \infty} d\left(x_{n}, x_{n+1}\right) \rightarrow 0
$$

If $\left\{x_{n}\right\}$ is not a $b$-Cauchy sequence, then there exists $\epsilon>0$ and two sequences of positive integers $\{m(k)\}$ and $\{n(k)\}$ with $n(k)>m(k) \geq k$ such that for all positive intiger $k, d\left(x_{m(k)}, x_{n(k)}\right) \geq \epsilon, d\left(x_{m(k)}, x_{n(k-1)}\right)<\epsilon$ and

(a) $\epsilon \leq \lim _{n \rightarrow \infty} \operatorname{infd}\left(x_{m(k)}, x_{n(k)}\right) \leq \lim _{n \rightarrow \infty} \operatorname{supd}\left(x_{m(k)}, x_{n(k)}\right) \leq s \epsilon$.

(b) $\frac{\epsilon}{s} \leq \lim _{n \rightarrow \infty} \operatorname{infd}\left(x_{m(k)}, x_{n(k)}\right) \leq \lim _{n \rightarrow \infty} \operatorname{supd}\left(x_{m(k+1)}, x_{n(k)}\right) \leq s^{2} \epsilon$.

(c) $\frac{\epsilon}{s_{\epsilon}^{2}} \leq \lim _{n \rightarrow \infty} \operatorname{infd}\left(x_{m(k+1)}, x_{n(k)}\right) \leq \lim _{n \rightarrow \infty} \operatorname{supd}\left(x_{m(k)}, x_{n(k+1)}\right) \leq s^{2} \epsilon$.

(d) $\frac{\epsilon}{s^{2}} \leq \lim _{n \rightarrow \infty} \operatorname{infd}\left(x_{m(k+1)}, x_{n(k+1)}\right) \leq \lim _{n \rightarrow \infty} \operatorname{supd}\left(x_{m(k+1)}, x_{n(k+1)}\right) \leq s^{3} \epsilon$

holds.

\section{Results and discussion}

In this section, we introduce a generalized weakly contractive mappings in the setting of $b$-metric spaces and prove a fixed point result.

Definition 5. Let $X$ be a $b$-metric space with metric $d$ and parameter $s \geq 1, T: X \rightarrow X$, and let $\varphi: X \rightarrow R^{+}$ be a lower semicontinuous function, then $T$ is called a generalized weakly contractive mapping if satisfies the following condition:

$$
\psi\left(S^{3} d(T x, T y)+\varphi(T x)+\varphi(T y)\right) \leq \psi(m(x, y, d, T, \varphi))-\phi(l(x, y, d, T, \varphi))
$$


for all $x, y \in X$, where,

$$
\begin{aligned}
m(x, y, d, T, \varphi)= & \max \{d(x, y)+\varphi(x)+\varphi(y), d(x, T x)+\varphi(x)+\varphi(T x), d(y, T y)+\varphi(y)+\varphi(T y), \\
& \left.\frac{1}{2 s^{2}}\{d(x, T y)+\varphi(x)+\varphi(T y)+d(y, T x)+\varphi(y)+\varphi(T x)\}\right\}
\end{aligned}
$$

and

$$
l(x, y, d, T, \varphi)=\max \{d(x, y)+\varphi(x)+\varphi(y), d(y, T y)+\varphi(y)+\varphi(T y)\}
$$

for all $x, y \in X$, where $\psi: R^{+} \rightarrow R^{+}$is a continuous with $\psi(t)=0$ if and only if $t=0$ and $\phi: R^{+} \rightarrow R^{+}$is a lower semicontinuous function with $\phi(t)=0$ if and only if $t=0$.

Theorem 2. Let $X$ be a complete b-metric space with metric $d$ and $s \geq 1$. If $T$ is a generalized weakly contractive mapping then $T$ has a unique fixed point $u \in X$ such that $u=T u$ and $\varphi(u)=0$.

Proof. Let $x_{0} \in X$ be fixed and define a sequence $\left\{x_{n}\right\}$ by $x_{1}=T x_{0}, x_{2}=T x_{1}, \ldots, x_{n+1}=T x_{n}$ for all $n=$ $0,1,2, \ldots$ If $x_{n}=x_{n+1}$ for some $n, x_{n}=x_{n+1}=T x_{n}, x_{n}$ is fixed point of $T$.

Assume $x_{n} \neq x_{n+1}$ for all $n=0,1,2, \ldots$. From (2) by using $x=x_{n-1}$ and $y=x_{n}$, we have $m\left(x_{n-1}, x_{n}, d, T, \varphi\right)=\max \left\{d\left(x_{n-1}, x_{n}\right)+\varphi\left(x_{n-1}\right)+\varphi\left(x_{n}\right), d\left(x_{n-1}, T x_{n-1}\right)+\varphi\left(x_{n-1}\right)+\varphi\left(T x_{n-1}\right), d\left(x_{n}, T x_{n}\right)+\right.$ $\left.\varphi\left(x_{n}\right)+\varphi\left(T x_{n}\right), \frac{1}{2 s^{2}}\left\{d\left(x_{n-1}, T x_{n}\right)+\varphi\left(x_{n-1}\right)+\varphi\left(T x_{n}\right)+d\left(x_{n}, T x_{n-1}\right)+\varphi\left(x_{n}\right)+\varphi\left(T x_{n-1}\right)\right\}\right\}=$ $\max \left\{d\left(x_{n-1}, x_{n}\right)+\varphi\left(x_{n-1}\right)+\varphi\left(x_{n}\right), d\left(x_{n-1}, x_{n}\right)+\varphi\left(x_{n-1}\right)+\varphi\left(x_{n}\right) d\left(x_{n}, x_{n+1}\right)+\varphi\left(x_{n}\right)+\right.$ $\left.\varphi\left(x_{n+1}\right), \frac{1}{2 s^{2}}\left\{d\left(x_{n-1}, x_{n+1}\right)+\varphi\left(x_{n-1}\right)+\varphi\left(x_{n+1}\right)+d\left(x_{n}, x_{n}\right)+\varphi\left(x_{n}\right)+\varphi\left(x_{n}\right)\right\}\right\}$.

Since

$\frac{1}{2 s^{2}}\left\{d\left(x_{n-1}, x_{n+1}\right)+\varphi\left(x_{n-1}\right)+\varphi\left(x_{n+1}\right)+d\left(x_{n}, x_{n}\right)+\varphi\left(x_{n}\right)+\varphi\left(x_{n}\right)\right\} \leq \frac{1}{2 s^{2}}\left\{s d\left(x_{n-1}, x_{n}\right)+\varphi\left(x_{n-1}\right)+\varphi\left(x_{n}\right)+\right.$ $\left.s d\left(x_{n}, x_{n+1}\right)+\varphi\left(x_{n}\right)+\varphi\left(x_{n+1}\right)\right\} \leq \frac{1}{2 s^{2}}\left\{s\left[d\left(x_{n-1}, x_{n}\right)+\varphi\left(x_{n-1}\right)+\varphi\left(x_{n}\right)+d\left(x_{n}, x_{n+1}\right)+\varphi\left(x_{n}\right)+\varphi\left(x_{n+1}\right)\right]\right\}=$ $\frac{1}{2 s}\left\{d\left(x_{n-1}, x_{n}\right)+\varphi\left(x_{n-1}\right)+\varphi\left(x_{n}\right)+d\left(x_{n}, x_{n+1}\right)+\varphi\left(x_{n}\right)+\varphi\left(x_{n+1}\right)\right\} \leq \frac{1}{2}\left\{d\left(x_{n-1}, x_{n}\right)+\varphi\left(x_{n-1}\right)+\varphi\left(x_{n}\right)+\right.$ $\left.d\left(x_{n}, x_{n+1}\right)+\varphi\left(x_{n}\right)+\varphi\left(x_{n+1}\right)\right\} \leq \max \left\{d\left(x_{n-1}, x_{n}\right)+\varphi\left(x_{n-1}\right)+\varphi\left(x_{n}\right), d\left(x_{n}, x_{n+1}\right)+\varphi\left(x_{n}\right)+\varphi\left(x_{n+1}\right)\right\}$.

So, we obtain

$$
m\left(x_{n-1}, x_{n}, d, T, \varphi\right)=\max \left\{d\left(x_{n-1}, x_{n}\right)+\varphi\left(x_{n-1}\right)+\varphi\left(x_{n}\right), d\left(x_{n}, x_{n+1}\right)+\varphi\left(x_{n}\right)+\varphi\left(x_{n+1}\right)\right\} .
$$

Similarly from (3)

$$
l\left(x_{n-1}, x_{n}, d, T, \varphi\right)=\max \left\{d\left(x_{n-1}, x_{n}\right)+\varphi\left(x_{n-1}\right)+\varphi\left(x_{n}\right), d\left(x_{n}, x_{n+1}\right)+\varphi\left(x_{n}\right)+\varphi\left(x_{n+1}\right)\right\} .
$$

Then (1) becomes

$$
\psi\left(s^{3} d\left(x_{n}, x_{n+1}\right)+\varphi\left(x_{n}\right)+\varphi\left(x_{n+1}\right) \leq \psi\left(m\left(x_{n-1}, x_{n}, d, T, \varphi\right)\right)-\phi\left(l\left(x_{n-1}, x_{n}, d, T, \varphi\right)\right) .\right.
$$

Now, if $d\left(x_{n-1}, x_{n}\right)+\varphi\left(x_{n-1}\right)+\varphi\left(x_{n}\right) \leq d\left(x_{n}, x_{n+1}\right)+\varphi\left(x_{n}\right)+\varphi\left(x_{n+1}\right)$, for some positive integer $n$ then (6) becomes

$\psi\left(s^{3} d\left(x_{n}, x_{n+1}\right)+\varphi\left(x_{n}\right)+\varphi\left(x_{n+1}\right) \leq \psi\left(d\left(x_{n}, x_{n+1}\right)+\varphi\left(x_{n}\right)+\varphi\left(x_{n+1}\right)\right)-\phi\left(d\left(x_{n}, x_{n+1}\right)+\varphi\left(x_{n}\right)+\varphi\left(x_{n+1}\right)\right)\right.$.

It follows $\psi\left(d\left(x_{n}, x_{n+1}\right)+\varphi\left(x_{n}\right)+\varphi\left(x_{n+1}\right)\right) \leq \psi\left(s^{3} d\left(x_{n}, x_{n+1}\right)+\varphi\left(x_{n}\right)+\varphi\left(x_{n+1}\right) \leq \psi\left(d\left(x_{n}, x_{n+1}\right)+\right.\right.$ $\left.\varphi\left(x_{n}\right)+\varphi\left(x_{n+1}\right)\right)-\phi\left(d\left(x_{n}, x_{n+1}\right)+\varphi\left(x_{n}\right)+\varphi\left(x_{n+1}\right)\right) \psi\left(d\left(x_{n}, x_{n+1}\right)+\varphi\left(x_{n}\right)+\varphi\left(x_{n+1}\right)\right)$, which is a contradiction. Thus,

$$
d\left(x_{n-1}, x_{n}\right)+\varphi\left(x_{n-1}\right)+\varphi\left(x_{n}\right)>d\left(x_{n}, x_{n+1}\right)+\varphi\left(x_{n}\right)+\varphi\left(x_{n+1}\right) .
$$

From (4), (5) and (7), we obtain

$$
m\left(x_{n-1}, x_{n}, d, T, \varphi\right)=l\left(x_{n-1}, x_{n}, d, T, \varphi\right)=d\left(x_{n-1}, x_{n}\right)+\varphi\left(x_{n-1}\right)+\varphi\left(x_{n}\right),
$$

So (6) becomes:

$$
\psi\left(s^{3} d\left(x_{n}, x_{n+1}\right)+\varphi\left(x_{n}\right)+\varphi\left(x_{n+1}\right) \leq \psi\left(m\left(x_{n-1}, x_{n}, d, T, \varphi\right)\right)-\phi\left(l\left(x_{n-1}, x_{n}, d, T, \varphi\right)\right) .\right.
$$


From (7), the sequence $\left(d\left(x_{n}, x_{n+1}\right)+\varphi\left(x_{n}\right)+\varphi\left(x_{n+1}\right)\right)$ is decreasing and bounded below. Hence $d\left(x_{n}, x_{n+1}\right)+\varphi\left(x_{n}\right)+\varphi\left(x_{n+1}\right) \rightarrow r$ as $n \rightarrow \infty$ for some $r \geq 0$. Assume $r>0$ and letting $n \rightarrow \infty$ in (9) and using the continuity of $\psi$ and the lower semicontinuity of $\phi$, we have

$$
\begin{aligned}
\psi\left(s^{3} r\right) & \leq \psi(r)-\lim _{n \rightarrow \infty} \inf \phi\left(d\left(x_{n-1}, x_{n}\right)+\varphi\left(x_{n-1}\right)+\varphi\left(x_{n}\right)\right) \\
& \leq \psi(r)-\lim _{n \rightarrow \infty} \phi\left(d\left(x_{n-1}, x_{n}\right)+\varphi\left(x_{n-1}\right)+\varphi\left(x_{n}\right)\right) \\
& =\psi(r)-\phi(r) .
\end{aligned}
$$

It follows that $\psi(r) \leq \psi\left(s^{3} r\right) \leq \psi(r)-\phi(r)<\psi(r)$, which is a contradiction, hence we have $r=0$ and consequently, $\lim _{n \rightarrow \infty}\left[d\left(x_{n}, x_{n+1}\right)+\varphi\left(x_{n}\right)+\varphi\left(x_{n+1}\right)\right]=0$. Implies

$$
\begin{aligned}
& \lim _{n \rightarrow \infty} d\left(x_{n}, x_{n+1}\right)=0 . \\
& \lim _{n \rightarrow \infty} \varphi\left(x_{n}\right)=\lim _{n \rightarrow \infty} \varphi\left(x_{n+1}\right)=0 .
\end{aligned}
$$

Now, we prove that the sequence $\left\{x_{n}\right\}$ is a $b$-Cauchy sequence. If $\left\{x_{n}\right\}$ is not a $b$-Cauchy sequence, then by Lemma 1 there exists $\epsilon>0$ and sequences of positive integers $m(k)$ and $n(k)$ such that for all positive integer $k, n(k)>m(k) \geq k, d\left(x_{m(k)}, x_{n(k)}\right) \geq \epsilon$ and $d\left(x_{m(k)}, x_{n(k-1)}\right)<\epsilon$ and conditions from (a)-(d) of 1 hold.

From (2) and by setting $x=x_{m(k)}$ and $y=x_{n(k)}$ we have:

$m\left(x_{m(k)}, x_{n(k)}, d, T, \varphi\right)=\max \left\{\left(d\left(x_{m(k)}, x_{n(k)}\right)+\varphi\left(x_{m(k)}\right)+\varphi\left(x_{n(k)}\right), d\left(x_{m(k)}, T x_{m(k)}\right)+\varphi\left(x_{m(k)}\right)+\right.\right.$ $\varphi\left(T x_{m(k)}\right), d\left(x_{n(k)}, T x_{n(k)}\right)+\varphi\left(x_{n(k)}\right)+\varphi\left(T x_{n(k)}\right), \frac{1}{2 s^{2}}\left\{d\left(x_{m(k)}, T x_{n(k)}\right)+\varphi\left(x_{m(k)}\right)+\varphi\left(T x_{n(k)}\right)+\right.$ $\left.\left.d\left(x_{n(k)}, T x_{m(k)}\right)+\varphi\left(x_{n(k)}\right)+\varphi\left(T x_{m(k)}\right)\right\}\right\}=\max \left\{d\left(x_{m(k)}, x_{n(k)}\right)+\varphi\left(x_{m(k)}\right)+\varphi\left(x_{n(k)}\right), d\left(x_{m(k)}, x_{m(k)+1}\right)+\right.$ $\varphi\left(x_{m(k)}\right)+\varphi\left(x_{m(k)+1}\right), d\left(x_{n(k)}, x_{n(k)+1}\right)+\varphi\left(x_{n(k)}\right)+\varphi\left(x_{n(k)+1}\right), \frac{1}{2 S^{2}}\left\{d\left(x_{m(k)}, x_{n(k)+1}\right)+\varphi\left(x_{m(k)}\right)+\varphi\left(x_{n(k)+1}\right)+\right.$ $\left.\left.d\left(x_{n(k)}, x_{m(k)+1}\right)+\varphi\left(x_{n(k)}\right)+\varphi\left(x_{m(k)+1}\right)\right\}\right\}$.

Taking the limit as $k \rightarrow \infty$ and using (10), (11) and Lemma 1, we have

$$
\begin{aligned}
& \lim _{k \rightarrow \infty} m\left(x_{m(k)}, x_{n(k)}, d, T, \varphi\right)=\max \lim _{k \rightarrow \infty}\left\{d\left(x_{m(k)}, x_{n(k)}\right)+\varphi\left(x_{m(k)}\right)+\varphi\left(x_{n(k)}\right), d\left(x_{m(k)}, x_{m(k)+1}\right)+\varphi\left(x_{m(k)}\right)\right. \\
& +\varphi\left(x_{m(k)+1}\right), d\left(x_{n(k)}, x_{n(k)+1}\right)+\varphi\left(x_{n(k)}\right)+\varphi\left(x_{n(k)+1}\right), \frac{1}{2 S^{2}}\left\{d\left(x_{m(k)}, x_{n(k)+1}\right)+\varphi\left(x_{m(k)}\right)+\varphi\left(x_{n(k)+1}\right)\right. \\
& \left.\left.+d\left(x_{n(k)}, x_{m(k)+1}\right)+\varphi\left(x_{n(k)}\right)+\varphi\left(x_{m(k)+1}\right)\right\}\right\} \leq \max \left\{s \epsilon, 0,0, \frac{1}{2 s^{2}}\left(s^{2} \epsilon+s^{2} \epsilon\right)\right\}=s \epsilon .
\end{aligned}
$$

Similarly from (3), we have

$l\left(x_{m(k)}, x_{n(k)}, d, T, \varphi\right)=\max \left\{d\left(x_{m(k)}, x_{n(k)}\right)+\varphi\left(x_{m(k)}\right)+\varphi\left(x_{n(k)}\right), d\left(x_{n(k)}, T x_{n(k)}\right)+\varphi\left(x_{n(k)}\right)+\varphi\left(T x_{n(k)}\right)\right\}=$ $\max \left\{d\left(x_{m(k)}, x_{n(k)}\right)+\varphi\left(x_{m(k)}\right)+\varphi\left(x_{n(k)}\right), d\left(x_{n(k)}, x_{n(k)+1}\right)+\varphi\left(x_{n(k)}\right)+\varphi\left(x_{n(k)+1}\right)\right\}$

$$
\begin{aligned}
& \lim _{k \rightarrow \infty} l\left(x_{m(k)}, x_{n(k)}, d, T, \varphi\right)=\lim _{k \rightarrow \infty} \max \left\{d\left(x_{m(k)}, x_{n(k)}\right)+\varphi\left(x_{m(k)}\right)+\varphi\left(x_{n(k)}\right), d\left(x_{n(k)}, x_{n(k)+1}\right)+\varphi\left(x_{n(k)}\right)\right. \\
& \left.+\varphi\left(x_{n(k)+1}\right)\right\} \leq \max \{s \epsilon, 0\}=s \epsilon .
\end{aligned}
$$

Now from (1), we have

$\psi\left(s^{3} d(T x, T y)+\varphi(T x)+\varphi(T y)\right)=\psi\left(s^{3} d\left(x_{m(k)+1}, x_{n(k)+1}\right)+\varphi\left(x_{m(k)+1}\right)+\varphi\left(x_{n(k)+1}\right)\right) \leq$ $\psi\left(m\left(x_{m(k)}, x_{n(k)}, d, T, \varphi\right)\right)-\phi\left(l\left(x_{m(k)}, x_{n(k)}, d, T, \varphi\right)\right)$.

Letting $k \rightarrow \infty$, using (11), (12), (13), applying the continuity of $\psi$ and lower semicontinuity of $\phi$, we have,

$$
\lim _{k \rightarrow \infty} \psi\left(s^{3} d\left(x_{m(k)+1}, x_{n(k)+1}\right)\right) \leq \psi(s \varepsilon)-\phi(s \epsilon) .
$$

This implies that

$$
\psi(s \epsilon)=\psi\left(s^{3} \frac{\varepsilon}{s^{2}}\right) \leq \psi\left(s^{3} \lim _{k \rightarrow \infty} d\left(x_{m(k)+1}, x_{n(k)+1}\right)\right) \leq \psi(s \varepsilon)-\phi(s \epsilon)<\psi(s \epsilon),
$$

which is a contradiction. Therefore $\left\{x_{n}\right\}$ is a $b$-Cauchy sequence. Now since $\left\{x_{n}\right\}$ is a $b$-Cauchy and $X$ is $b$-complete we have,

$$
\lim _{n \rightarrow \infty} x_{n}=u \in X
$$


Since $\varphi$ is lower semicontinuous,

$$
\varphi(u) \leq \lim _{n \rightarrow \infty} \inf \varphi\left(x_{n}\right) \leq \lim _{n \rightarrow \infty} \varphi\left(x_{n}\right)=0,
$$

which implies

$$
\varphi(u)=0
$$

Now from (2) by putting $x=x_{n}$ and $y=u$, we have

$m\left(x_{n}, u, d, T, \varphi\right)=\max \left\{d\left(x_{n}, u\right)+\varphi\left(x_{n}\right)+\varphi(u), d\left(x_{n}, T x_{n}\right)+\varphi\left(x_{n}\right)+\varphi\left(T x_{n}\right), d(u, T u)+\varphi(u)+\right.$ $\left.\varphi(T u), \frac{1}{2 s^{2}}\left\{d\left(x_{n}, T u\right)+\varphi\left(x_{n}\right)+\varphi(T u)+d\left(u, T x_{n}\right)+\varphi(u)+\varphi\left(T x_{n}\right)\right\}\right\}=\max \left\{d\left(x_{n}, u\right)+\varphi\left(x_{n}\right)+\right.$ $\varphi(u), d\left(x_{n}, x_{n+1}\right)+\varphi\left(x_{n}\right)+\varphi\left(x_{n+1}\right), d(u, T u)+\varphi(u)+\varphi(T u), \frac{1}{2 s^{2}}\left\{d\left(x_{n}, T u\right)+\varphi\left(x_{n}\right)+\varphi(T u)+d\left(u, x_{n+1}\right)+\right.$ $\left.\left.\varphi(u)+\varphi\left(x_{n+1}\right)\right\}\right\}$.

Applying the limit as $n \rightarrow \infty$ and using (10), (11) and(14) we have

$$
\lim _{n \rightarrow \infty} m\left(x_{n}, u, d, T, \varphi\right)=d(u, T u)+\varphi(T u) .
$$

Similarly

$$
\lim _{n \rightarrow \infty} l\left(x_{n}, u, d, T, \varphi\right)=d(u, T u)+\varphi(T u) .
$$

Then using (1), we have

$$
\begin{aligned}
\psi\left(s^{3} d\left(T x_{n}, T u\right)+\varphi\left(T x_{n}\right)+\varphi(T u)\right) & =\psi\left(s^{3} d\left(x_{n+1}, T u\right)+\varphi\left(x_{n+1}\right)+\varphi(T u)\right) \\
& \leq \psi\left(m\left(x_{n}, u, d, T, \varphi\right)\right)-\phi\left(l\left(x_{n}, u, d, T, \varphi\right)\right) .
\end{aligned}
$$

Letting $n \rightarrow \infty$, using (14),(15), (16) and by using the continuity of $\psi$ and lower semicontinuity of $\phi$, we have

$$
\begin{aligned}
\psi\left(s^{3} d\left(x_{n+1}, T u\right)+\varphi\left(x_{n+1}\right)+\varphi(T u)\right) & =\psi\left(s^{3} d(u, T u)+\varphi(T u)\right) \\
& \leq \psi\left(m\left(x_{n}, u, d, T, \varphi\right)\right)-\phi\left(l\left(x_{n}, u, d, T, \varphi\right)\right) \\
& =\psi(d(u, T u)+\varphi(T u))-\phi(d(u, T u)+\varphi(T u)) .
\end{aligned}
$$

This implies

$$
\begin{aligned}
\psi(d(u, T u)+\varphi(T u)) & \leq \psi\left(s^{3} d(u, T u)+\varphi(T u)\right) \\
& \leq \psi(d(u, T u)+\varphi(T u))-\phi(d(u, T u)+\varphi(T u)) .
\end{aligned}
$$

This holds if and only if, $\phi(d(u, T u)+\varphi(T u))=0$ and then from the property of $\phi$ we have,

$$
d(u, T u)+\varphi(T u)=0 .
$$

Hence, $d(u, T u)=0$ so that $\mathrm{u}=\mathrm{Tu}$ and $\varphi(T u)=0$. Since $u=T u$ this implies $\varphi(u)=0$.

Therefore $u$ is fixed point of $T$.

\section{Uniqueness}

Suppose $v$ is another fixed point of $T$. Then $T v=v$ and $\varphi(v)=0$.

By (1) with $x=u$ and $y=v$

$$
\psi\left(s^{3} d(T u, T v)+\varphi(T u)+\varphi(T v)\right)=\psi\left(s^{3} d(u, v)\right) \leq \psi(m(T u, T v, d, T, \varphi))-\phi(l(T u, T v, d, T, \varphi)) .
$$

From (2) we have $m(T u, T v, d, T, \varphi)=\max \left\{\left(d(T u, T v)+\varphi(T u)+\varphi(T v), d\left(T u, T^{2} u\right)+\varphi(T u)+\varphi\left(T^{2} u\right), d\left(T v, T^{2} v\right)+\varphi(T v)+\right.\right.$ $\left.\varphi\left(T^{2} v\right), \frac{1}{2 s^{2}}\left\{d\left(T u, T^{2} v\right)+\varphi(T u)+\varphi\left(T^{2} v\right)+d\left(T v, T^{2} u\right)+\varphi(T v)+\varphi\left(T^{2} u\right)\right\}\right\}=\max \{(d(u, v)+\varphi(u)+$ $\left.\varphi(v), d(u, u)+\varphi(u)+\varphi(u), d(v, v)+\varphi(v)+\varphi(v), \frac{1}{2 s^{2}}\{d(u, v)+\varphi(u)+\varphi(v)+d(v, u)+\varphi(v)+\varphi(u)\}\right\}=$ $d(u, v)$.

Similarly from (3), we have 


$$
\begin{aligned}
l(T u, T v, d, T, \varphi) & =\max \left\{d(T u, T v)+\varphi(T u)+\varphi(T v), d\left(T v, T^{2} v\right)+\varphi(T v)+\varphi\left(T^{2} v\right)\right. \\
& =d(u, v)+\varphi(u)+\varphi(v), d(v, v)+\varphi(v)+\varphi(v)\}=d(u, v) .
\end{aligned}
$$

Then using (1) and the continuity of $\psi$, we have

$$
\psi(d(u, v)) \leq \psi\left(s^{3} d(u, v)\right) \leq \psi(d(u, v))-\phi(d(u, v))
$$

This holds if $\phi(d(u, v))=0$ and then we have $d(u, v)=0$. Hence $u=v$. Therefore, $T$ has a unique fixed point.

Example 2. Let $X=[0,1]$ and $d(x, y)=(x-y)^{2}$. Then $(X, d)$ is $b$-metric space with $s=2$. Define $T: X \rightarrow X$, $\varphi: X \rightarrow R^{+}$and $\psi, \phi: R^{+} \rightarrow R^{+}$by $T(x)=\left\{\begin{array}{ll}0 & \text { if } x \leq \frac{1}{4} \\ \frac{1}{16} & \text { if } x \in\left(\frac{1}{4}, 1\right]^{\prime}\end{array}, \psi(t)=\frac{5 t}{4}, \phi(t)=\left\{\begin{array}{ll}\frac{t}{8} & \text { if } t \leq 3 \\ \frac{t}{4} & \text { if } t>3\end{array}\right.\right.$ and $\varphi(t)=\left\{\begin{array}{ll}2 t & \text { if } t>1 \\ t & \text { if } 0 \leq t \leq 1\end{array}\right.$. Now we verify condition (1).

Case I: If $x, y \in\left[0, \frac{1}{4}\right]$ and $x \geq y$. Then $\psi\left[s^{3} d(T x, T y)+\varphi(T x)+\varphi(T y)\right]=\psi\left[2^{3}(T x-T y)^{2}+\varphi(T x)+\varphi(T y)\right]=$ $\psi[8(0)+\varphi(0)+\varphi(0)]=0$.

Also $d(x, y)+\varphi(x)+\varphi(y)=(x-y)^{2}+\varphi(x)+\varphi(y)=(x-y)^{2}+x+y$,

$d(x, T x)+\varphi(x)+\varphi(T x)=(x-T x)^{2}+\varphi(x)+\varphi(T x)=x^{2}+x$,

$d(y, T y)+\varphi(y)+\varphi(T y)=(y-T y)^{2}+\varphi(y)+\varphi(T y)=y^{2}+y$,

$\frac{1}{s^{2}}[d(x, T y)+\varphi(x)+\varphi(T y)+d(y, T x)+\varphi(y)+\varphi(T x)]=\frac{1}{8}\left(x^{2}+x+y^{2}+y\right)$.

And $m(x, y, d, T, \varphi)=\max \left\{(x-y)^{2}+x+y, x^{2}+x, y^{2}+y, \frac{1}{8}\left(x^{2}+x+y^{2}+y\right)\right\}$,

$\frac{1}{8}\left(x^{2}+x+y^{2}+y\right) \leq \max \left\{x^{2}+x, y^{2}+y\right\}=x^{2}+x$,

$m(x, y, d, T, \varphi)=\max \left\{(x-y)^{2}+x+y, x^{2}+x\right\}$

and $l(x, y, d, T, \varphi)=\max \left\{(x-y)^{2}+x+y, y^{2}+y\right\}$.

But $(x-y)^{2}+x+y \geq x+y \geq y+y \geq y^{2}+y$, so,$l(x, y, d, T, \varphi)=(x-y)^{2}+x+y$ and $\phi[l(x, y, d, T, \varphi)]=$ $\frac{1}{8}\left[(x-y)^{2}+x+y\right]$.

If $m(x, y, d, T, \varphi)=(x-y)^{2}+x+y$ then (1) becomes $0 \leq \frac{5}{4}\left[(x-y)^{2}+x+y\right]-\frac{1}{8}\left[(x-y)^{2}+x+y\right]=$ $\frac{9}{8}\left[(x-y)^{2}+x+y\right]$ and if $m(x, y, d, T, \varphi)=x^{2}+x$ then by $(1)$, we have $\frac{5}{4}\left(x^{2}+x\right)-\frac{1}{8}\left[(x-y)^{2}+x+y\right] \geq$ $\frac{5}{4}\left(x^{2}+x\right)-\frac{1}{8}\left(x^{2}+x\right)=\frac{9}{8}\left(x^{2}+x\right) \geq 0$.

Let $x, y \in\left[0, \frac{1}{4}\right]$ and $x<y$. Then $m(x, y, d, T, \varphi)=\max \left\{(x-y)^{2}+x+y, x^{2}+x, y^{2}+y, \frac{1}{8}\left(x^{2}+x+y^{2}+y\right)\right\}$, $\frac{1}{8}\left(x^{2}+x+y^{2}+y\right) \leq \max \left\{x^{2}+x, y^{2}+y\right\}=y^{2}+y$, implies $m(x, y, d, T, \varphi)=\max \left\{(x-y)^{2}+x+y, y^{2}+y\right\}$.

Similarly $l(x, y, d, T, \varphi)=\max \left\{(x-y)^{2}+x+y, y^{2}+y\right\}$. Now, if $m(x, y, d, T, \varphi)=(x-y)^{2}+x+y=$ $l(x, y, d, T, \varphi),(1)$ becomes $0 \leq \frac{9}{8}\left[(x-y)^{2}+x+y\right]$. If $m(x, y, d, T, \varphi)=y^{2}+y=l(x, y, d, T, \varphi)$, (1) becomes $0 \leq \frac{9}{8}\left(y^{2}+y\right)$.

Case II: If $x \in\left[0, \frac{1}{4}\right], y \in\left(\frac{1}{4}, 1\right]$. This implies $x<y$. Then $\psi\left[s^{3} d(T x, T y)+\varphi(T x)+\varphi(T y)\right]=\psi\left[2^{3}(T x-T y)^{2}+\right.$ $\varphi(T x)+\varphi(T y)]=\frac{5}{4}\left[8\left(0-\frac{1}{16}\right)^{2}+\varphi(0)+\varphi\left(\frac{1}{16}\right)\right]=\frac{5}{4}\left(\frac{1}{32}+\frac{1}{16}\right)=\frac{15}{128}$.

Also $d(x, y)+\varphi(x)+\varphi(y)=(x-y)^{2}+\varphi(x)+\varphi(y)=(x-y)^{2}+x+y$, $d(x, T x)+\varphi(x)+\varphi(T x)=(x-T x)^{2}+\varphi(x)+\varphi(T x)=x^{2}+x$, $d(y, T y)+\varphi(y)+\varphi(T y)=\left(y-\frac{1}{16}\right)^{2}+y+\frac{1}{16}=y^{2}+\frac{7 y}{8}+\frac{17}{256}$ $\frac{1}{s^{2}}[d(x, T y)+\varphi(x)+\varphi(T y)+d(y, T x)+\varphi(y)+\varphi(T x)]=\frac{1}{8}\left[x^{2}+\frac{7 x}{8}+y^{2}+y+\frac{17}{256}\right]$.

And $m(x, y, d, T, \varphi)=\max \left\{(x-y)^{2}+x+y, x^{2}+x, y^{2}+\frac{7 y}{8}+\frac{17}{256}, \frac{1}{8}\left[x^{2}+\frac{7 x}{8}+y^{2}+y+\frac{17}{256}\right]\right\}$, $\frac{1}{8}\left[x^{2}+\frac{7 x}{8}+y^{2}+y+\frac{17}{256}\right]=\frac{1}{4}\left[\frac{x^{2}}{2}+\frac{7 x}{16}+\frac{y^{2}}{2}+\frac{y}{2}+\frac{17}{512}\right] \leq \frac{1}{4}\left[x^{2}+x+y^{2}+\frac{7 y}{8}+\frac{17}{256}\right] \leq \max \left\{x^{2}+x, y^{2}+\frac{7 y}{8}+\right.$ $\left.\frac{17}{256}\right\}=y^{2}+\frac{7 y}{8}+\frac{17}{256}$.

Therefore $m(x, y, d, T, \varphi)=\max \left\{(x-y)^{2}+x+y, y^{2}+\frac{7 y}{8}+\frac{17}{256}\right\}$. Similarly, $l(x, y, d, T, \varphi)=\max \{(x-$ $\left.y)^{2}+x+y, y^{2}+\frac{7 y}{8}+\frac{17}{256}\right\}$.

Now if, $m(x, y, d, T, \varphi)=(x-y)^{2}+x+y=l(x, y, d, T, \varphi)$, then $\psi[m(x, y, d, T, \varphi)]=\frac{5}{4}\left[(x-y)^{2}+x+y\right]$ and $\phi[m(x, y, d, T, \varphi)]=\frac{1}{8}\left[(x-y)^{2}+x+y\right]$. So (1) becomes $\frac{15}{128} \leq \frac{9}{8}\left[(x-y)^{2}+x+y\right]$.

Also if, $m(x, y, d, T, \varphi)=y^{2}+\frac{7 y}{8}+\frac{17}{256}=l(x, y, d, T, \varphi)$, then $\psi[m(x, y, d, T, \varphi)]=\frac{5}{4}\left[y^{2}+\frac{7 y}{8}+\frac{17}{256}\right]$ and $\phi[m(x, y, d, T, \varphi)]=\frac{1}{8}\left[y^{2}+\frac{7 y}{8}+\frac{17}{256}\right]$. So (1) becomes $\frac{15}{128} \leq \frac{9}{8}\left[y^{2}+\frac{7 y}{8}+\frac{17}{256}\right]$. 
Case III: If $x, y \in\left(\frac{1}{4}, 1\right]$ and $x \geq y$. Then $\psi\left[s^{3} d(T x, T y)+\varphi(T x)+\varphi(T y)\right]=\psi\left[8\left(\frac{1}{16}-\frac{1}{16}\right)^{2}+\frac{1}{16}+\frac{1}{16}\right]=\psi\left[\frac{1}{8}\right]=$ $\frac{5}{32}$.

Also $d(x, y)+\varphi(x)+\varphi(y)=(x-y)^{2}+x+y, d(x, T x)+\varphi(x)+\varphi(T x)=\left(x-\frac{1}{16}\right)^{2}+x+\frac{1}{16}$, $d(y, T y)+\varphi(y)+\varphi(T y)=\left(y-\frac{1}{16}\right)^{2}+y+\frac{1}{16}$,

$\left.\frac{1}{s^{2}}[d(x, T y)+\varphi(x)+\varphi(T y)+d(y, T x)+\varphi(y)+\varphi(T x)]=\frac{1}{8}\left[\left(x-\frac{1}{16}\right)^{2}+x+\frac{1}{16}+\left(y-\frac{1}{16}\right)^{2}+y+\frac{1}{16}\right)\right] \leq$ $\max \left[\left(x-\frac{1}{16}\right)^{2}+x+\frac{1}{16},\left(y-\frac{1}{16}\right)^{2}+y+\frac{1}{16}\right]=\left(x-\frac{1}{16}\right)^{2}+x+\frac{1}{16}$.

This implies that $m(x, y, d, T, \varphi)=\max \left\{(x-y)^{2}+x+y,\left(x-\frac{1}{16}\right)^{2}+x+\frac{1}{16}\right\}$ and $l(x, y, d, T, \varphi)=$ $\max \left\{(x-y)^{2}+x+y,\left(y-\frac{1}{16}\right)^{2}+y+\frac{1}{16}\right\}$. But $\left(y-\frac{1}{16}\right)^{2}+y+\frac{1}{16}=\left(\frac{1}{16}-y\right)^{2}+y+\frac{1}{16}<(x-y)^{2}+y+x$. So $l(x, y, d, T, \varphi)=(x-y)^{2}+x+y$.

Now if, $m(x, y, d, T, \varphi)=(x-y)^{2}+x+y$ (1) becomes, $\frac{5}{32} \leq \frac{5}{4}\left[(x-y)^{2}+x+y\right]-\frac{1}{8}\left[(x-y)^{2}+x+y\right]=$ $\frac{9}{8}\left[(x-y)^{2}+x+y\right]$.

If $m(x, y, d, T, \varphi)=\left(x-\frac{1}{16}\right)^{2}+x+\frac{1}{16} \quad$ then we have, $\frac{5}{4}\left[\left(x-\frac{1}{16}\right)^{2}+x+\frac{1}{16}\right]-\frac{1}{8}\left[(x-y)^{2}+x+y\right]>$ $\frac{5}{4}\left[\left(x-\frac{1}{16}\right)^{2}+x+\frac{1}{16}\right]-\frac{1}{8}\left[\left(x-\frac{1}{16}\right)^{2}+x+\frac{1}{16}\right]=\frac{9}{8}\left[\left(x-\frac{1}{16}\right)^{2}+x+\frac{1}{16}\right]>\frac{5}{32}$.

Let $x, y \in\left(\frac{1}{4}, 1\right]$ and $x<y$. Then $m(x, y, d, T, \varphi)=\max \left\{(x-y)^{2}+x+y,\left(y-\frac{1}{16}\right)^{2}+y+\frac{1}{16}\right\}=$ $l(x, y, d, T, \varphi)$.

Now if $m(x, y, d, T, \varphi)=l(x, y, d, T, \varphi)=(x-y)^{2}+x+y$, then (1) becomes $\frac{5}{32} \leq \frac{5}{4}\left[(x-y)^{2}+x+y\right]-$ $\frac{1}{8}\left[(x-y)^{2}+x+y\right]=\frac{9}{8}\left[(x-y)^{2}+x+y\right]$.

If $m(x, y, d, T, \varphi)=l(x, y, d, T, \varphi)=\left(y-\frac{1}{16}\right)^{2}+y+\frac{1}{16},(1)$ becomes $\frac{5}{32} \leq \frac{5}{4}\left[\left(y-\frac{1}{16}\right)^{2}+y+\frac{1}{16}\right]-\frac{1}{8}[(y-$ $\left.\left.\frac{1}{16}\right)^{2}+y+\frac{1}{16}\right]=\frac{9}{8}\left[\left(y-\frac{1}{16}\right)^{2}+y+\frac{1}{16}\right]$.

Case IV: If $x \in\left(\frac{1}{4}, 1\right], y \in\left[0, \frac{1}{4}\right]$. This implies $x>y$. Then $\psi\left[s^{3} d(T x, T y)+\varphi(T x)+\varphi(T y)\right]=\psi\left[2^{3}(T x-T y)^{2}+\right.$ $\varphi(T x)+\varphi(T y)]=\frac{5}{4}\left[8\left(\frac{1}{16}-0\right)^{2}+\varphi\left(\frac{1}{16}\right)++\varphi(0)\right]=\frac{5}{4}\left(\frac{1}{32}+\frac{1}{16}\right)=\frac{15}{128}$.

Also $d(x, y)+\varphi(x)+\varphi(y)=(x-y)^{2}+x+y$,

$d(x, T x)+\varphi(x)+\varphi(T x)=x^{2}+\frac{7 x}{8}+\frac{17}{256}$

$d(y, T y)+\varphi(y)+\varphi(T y)=y^{2}+y$,

$\frac{1}{s^{2}}[d(x, T y)+\varphi(x)+\varphi(T y)+d(y, T x)+\varphi(y)+\varphi(T x)]=\frac{1}{8}\left[x^{2}+x+y^{2}+\frac{7 y}{8}+\frac{17}{256}\right]$.

And $m(x, y, d, T, \varphi)=\max \left\{(x-y)^{2}+x+y, x^{2}+\frac{7 x}{8}+\frac{17}{256}, y^{2}+y, \frac{1}{8}\left[x^{2}+x+y^{2}+\frac{7 y}{8}+\frac{17}{256}\right]\right\}$,

$m(x, y, d, T, \varphi)=\max \left\{(x-y)^{2}+x+y, x^{2}+\frac{7 x}{8}+\frac{17}{256}\right\}$. Similarly, $l(x, y, d, T, \varphi)=\max \left\{(x-y)^{2}+x+y, y^{2}+\right.$ $y\}=(x-y)^{2}+x+y$.

Now if, $m(x, y, d, T, \varphi)=(x-y)^{2}+x+y=ł(x, y, d, T, \varphi)$, then (1) becomes $\frac{15}{128} \leq \frac{9}{8}\left[(x-y)^{2}+x+y\right]$. If, $m(x, y, d, T, \varphi)=x^{2}+\frac{7 x}{8}+\frac{17}{256}$, we have $\frac{5}{4}\left[x^{2}+\frac{7 x}{8}+\frac{17}{256}\right]-\frac{1}{8}\left[(x-y)^{2}+x+y\right]>\frac{5}{4}\left[x^{2}+\frac{7 x}{8}+\frac{17}{256}\right]-\frac{1}{8}\left[x^{2}+\right.$ $\left.\frac{7 x}{8}+\frac{17}{256}\right]=\frac{9}{8}\left[x^{2}+\frac{7 x}{8}+\frac{17}{256}\right]>\frac{15}{128}$.

Then (1) becomes $\frac{15}{128} \leq \frac{9}{8}\left[x^{2}+\frac{7 x}{8}+\frac{17}{256}\right]$. Thus all the condition of Theorem (2) are satisfied and 0 is the unique fixed point of $T$.

Remark 1. If we take $s=1$ in Theorem (2) we get the result of Cho [1]. Hence Our result generalizes the result of Cho [1] and related results in the literature.

Acknowledgments: The authors would like to thank Jimma University for material support.

Author Contributions: All authors contributed equally to the writing of this paper. All authors read and approved the final manuscript.

Conflicts of Interest: "The authors declare no conflict of interest."

\section{References}

[1] Cho, S. (2018). Fixed point theorems for generalized weakly contractive mappings in metric spaces with applications. Fixed point theory and application, 3, 1-18.

[2] Czerwik, S. (1993). Contraction mappings in b-metric spaces. Acta Mathematica et Informatica Universitatis Ostraviensis, 1(1), 5-11.

[3] Babu, G. V. R., \& Dula, T. M. (2018). Fixed points of almost generalized $(\alpha, \beta)-(\psi, \varphi)$-contractive mappings in $b$-metric spaces. Facta Universitatis, Series: Mathematics and Informatics, 33(2), 177-196.

[4] Aghajani, A., Abbas, M., \& Roshan, J. (2014). Common fixed point of generalized weak contractive mappings in partially ordered b-metric spaces. Mathematica Slovaca, 64(4), 941-960.

[5] Boriceanu, M. (2009). Strict fixed point theorems for multivalued operators in b-metric spaces. Internarional Journa of Modern Mathathematics, 4(3), 285-301. 
[6] Boriceanu, M., Bota, M., \& Petrusel, A. (2010). Multivalued fractals in b-metric spaces. Central European Journal of Mathematics, 8(2), 367-377.

[7] Boriceanu, M. (2009). Fixed point theory for multivalued generalized contraction on a set with two b-metrics. Studia Universitatis Babes-Bolyai, Mathematica, (3).

[8] Faraji, H., \& Nourouzi, K. (2017). Fixed and common fixed points for $(\psi, \varphi)$-weakly contractive mappings in $b$-metric spaces. Sahand Communications in Mathematical Analysis, 7(1), 49-62.

[9] Alber, Y. I., \& Guerre-Delabriere, S. (1997). Principle of weakly contractive maps in Hilbert spaces. In New results in operator theory and its applications (pp. 7-22). Birkhäuser, Basel.

[10] Rhoades, B. E. (2001). Some theorems on weakly contractive maps. Nonlinear Analysis: Theory, Methods E Applications, 47(4), 2683-2693.

[11] Choudhury, B. S., Konar, P., Rhoades, B. E., \& Metiya, N. (2011). Fixed point theorems for generalized weakly contractive mappings. Nonlinear Analysis: Theory, Methods \& Applications, 74(6), 2116-2126.

[12] Roshan, J. R., Parvaneh, V., \& Kadelburg, Z. (2014). Common fixed point theorems for weakly isotone increasing mappings in ordered b-metric spaces. Journal of Nonlinear Sciences and Applications, 7(4), 229-245. distributed under the terms and conditions of the Creative Commons Attribution (CC-BY) license (http://creativecommons.org/licenses/by/4.0/). 